\title{
SOURCE LOCALIZATION AND SENSOR PLACEMENT IN ENVIRONMENTAL MONITORING
}

\author{
ALEXANDER KHAPALOV \\ Department of Mathematics \\ Washington State University, Pullman, WA 99164-3113, USA \\ e-mail: khapala@wsu .edu
}

\begin{abstract}
In this paper we discuss two closely related problems arising in environmental monitoring. The first is the source localization problem linked to the question How can one find an unknown "contamination source"? The second is an associated sensor placement problem: Where should we place sensors that are capable of providing the necessary "adequate data" for that? Our approach is based on some concepts and ideas developed in mathematical control theory of partial differential equations.
\end{abstract}

Keywords: source localization, sensor placement, environmental monitoring, controllability, identifiability, parabolic equations.

\section{Introduction}

A typical problem associated with environmental monitoring of hazardous materials can, in general terms, be described as follows.

Let us assume that a certain area (it may be a water or groundwater basin or the atmosphere) is monitored by a number of sensors located in various sites. The sensors are used to evaluate the level of pollution in the area. Suppose that the incoming signals (measurement data) begin to inform one of an unexpected increase in the concentration of the pollutant, for example, owing to an unregistered accident, spill, etc. In a situation like this, one is interested in localizing as quickly as possible the new unknown source of contamination. We will further regard this problem as the source localization problem.

Clearly, one cannot succeed in solving the source localization problem if the measurement data are insufficient. This gives rise to the question on where to place sensors so that they can provide the necessary "adequate" data. This problem is further regarded as the sensor placement problem.

In this paper we discuss the theoretic foundations for the source localization and sensor placement problems, making use of the formalization involving diffusion system modeling, see, e.g., the works of Seinfeld (1986); Shukla et al. (1987); Dagan (1989); Demetriou (2009); Kurzhanski and Khapalov (1990) or Devooght and Smidts
(1996), and the references therein, as well as suitable concepts and ideas developed in mathematical controllability theory along the approach introduced by Khapalov (1994b). The focus of our findings is specifically on the issue of localization of the unknown sources (i.e., not on their precise description as, say, unknown initial conditions or forcing terms for the associated PDE as is normally required in a typical inverse problem).

A rather similar control-theoretic approach, namely, connecting the source identification (i.e., not necessarily localization) problem to controllability theory via duality, was used by Puel and Yamamoto (1995; 2005), Yamamoto (1995), Komornik and Yamamoto (2002), Nicaise and Zair (2004), Wang (2007) or Alvez et al. (2009) for the sources which are modeled as a partially unknown additive point or locally distributed controls of a particular form $\lambda(t) f(x)$, where the source intensity $\lambda(t)$ is assumed to be known, while its spatial distribution $f(x)$ is to be determined. The latter can be a $\delta$-function (point source) or, say, an element of a $L^{2}(\Omega)$ or $H_{0}^{1}(\Omega)$. In particular, such problems for the wave equation were considered in the works of Puel and Yamamoto (1995), Yamamoto (1995), and by Komornik and Yamamoto (2002; 2005), who also considered the heat equation; for the beam equation by Nicaise and Zair (2004), and for the plate equations by Wang (2007) and Alves et al. (2009). The main results of the above-cited works deal with establishing a one-to-one correspondence (and its stability) between the available 
data (typically infinite dimensional in these works such as, e.g., a trace of solution on the boundary) and the spatial distribution of the unknown source $f(x)$.

Contrary to the above, as in the work of Khapalov (1994b), in this paper we assume that the unknown source is the initial datum of special form for the process described by the parabolic PDE, in general, with time-dependent coefficients. The intensity of this source is assumed to be known as in the above-cited works. (A discussion on timedependent sources is given in Section 5 below as well.)

In a 1-D setup we consider the point models for unknown sources and for static and mobile sensors. We would like to emphasize that the point sensors provide finitedimensional (i.e., "practical" in real world applications) measurement data. We particularly exploit the advantages of the mobile point sensors whose data were proven to be stable to small errors in their locations-unlike their static counterparts (Khapalov, 2001; 1994a; 1995; 1998). Among early references on mobile point actuators and sensors, we refer the reader to the work of Butkovskii and Pustylnikov (1987) and the early references therein, and (Dolecki, 1973).

In the case of several dimensions, we consider the so-called "zone" (space-averaging) models of sources and sensors whose spatial distributions are described by the characteristic functions of some "small neighborhoods." (In other words, we assume that unknown sources were initiated in such neighborhoods.) Note that the zone sensors provide finite dimensional output as well.

Our approach is specifically designed for the aforementioned types of sources' supports (see, e.g., an example in Subsection 2.5 below). Our main interest is not as much in just establishing the aforementioned one-to-one correspondence between the data and source location. It is rather in finding out how one can set up a suitable practical strategy to find those sources as quickly as possible. To this end, our approach centers around the method of the so-called test-functions, introduced by Khapalov (1994b). In the framework of this method, we intend to derive certain algebraic equations of type $\gamma\left(x_{0}\right)=A$, where $x_{0}$ is the location of an unknown source, $\gamma(\cdot)$ is a suitable "easy test-function" and the value of $A$ is ("easily") determined by the actual measurement data. In a 1- $D$ case, we will attempt, for example, to construct a monotonic (at least on some interval of interest) test function, based solely on the structure of the PDE at hand and the location of the available point sensor(s). Thus, this method allows one to separate the "off-line" and "on-line" calculations, which can be critical in real world applications. (It should be noted along these lines that the numerical aspects of these calculation may pose a challenging related problem on their own.) The crux of this approach is in establishing the fact that one is able to construct a "good" suitable set of test functions, needed to identify the location of the unknown source, regardless of the measurement data that may occur. Of course, this will lead us to the aforementioned sensor placement problem.

Note that the goal of the source localization problem in our setup is to determine the position $x_{0}$ of the unknown source's support within the given bounded space domain. It is a nonlinear problem and therefore the standard controllability/observability duality approach does not apply directly in this case. (In this respect, in the above-cited works the unknown source was treated as an additive control from a suitable linear (i.e., unbounded) function space, and, thus, not always of "small" support.)

The above-outlined source localization problem can also be viewed as an inverse problem for partial differential equations with the goal to determine an unknown initial datum or a forcing term, see, e.g., the work of Isakov (2009) and the references therein. For other approaches to this issue, again with sources acting in time as unknown additive controls, we refer, e.g., to the research by Afifi $(2000 ; 2001)$, dealing with the source identification problem within a given subdomain or on the boundary (the regional detection); to the work of Demetriou (2009), where the author considered a closed loop system which simultaneously detects and suppresses the effect of unknown sources; and to the research of Matthes et al. (2005), dealing with numerical aspects of source localization problem in the case when the emitted substance is transported by advection caused by a known homogeneous wind field and by isotropic diffusion; and the references therein. For the probabilistic setting, we refer the reader to the research of Uciński (2000; 2005), who addresses a wider parameter identification problem, as well as Tzanos and Zefran (2006), and to the references therein.

We also refer to the interesting paper by Sivergina $e t$ al. (2003), where regularization procedures and recursive estimation algorithms were developed to estimate the location and the intensity of the source in systems modeled by parabolic equations.

The article is organized as follows. In Section 2 we consider a very simple model described by the standard diffusion/heat equation, assuming that we have just one point source acting at one instant of time only. We give a rigorous mathematical formulation of source localization and sensor placement problems, and describe our method of test-functions in great detail.

In Section 3 we extend the discussion to a rather general one dimensional reaction-diffusion-convection equation and focus on the principal difficulties arising in this case. These will lead us to the concepts of controllability and observability, which are introduced (or rather recalled) in Section 4. In Section 5, on a simple example we show how the method of test-functions can be applied to the models of sources which act continuously in time (i.e., with point sources) as in the works of Puel and Yamamoto (1995), Yamamoto (1995), Komornik and Yamamoto (2002; 2005), Nicaise and Zair (2004), Wang (2007) and 
Alvez et al., (2009). In Section 6 we extend the previous results to general multidimensional models.

\section{2. "Very simple" 1-D linear model with one point source and one point sensor}

2.1. Heat equation equation. Let us consider a diffusion process generated in the space-interval $(0,1)$ by a single point source of unit intensity (see Remark 1 for otherwise) located at an unknown point $x^{0} \in(0,1)$. Suppose that the evolution of this process during the time-interval $(0, T)$ is described by the following homogeneous Dirichlet boundary problem for the standard heat equation:

$$
\begin{gathered}
u_{t}=u_{x x} \quad \text { in }(0,1) \times(0, T)=Q_{T}, \\
\left.u\right|_{x=0,1}=0, \quad u(x, 0)=u_{0}(x)=\delta\left(x-x^{0}\right) .
\end{gathered}
$$

Here $u(x, t)$ is the concentration of a substance at point $x$ at time $t$.

Clearly, the solution to the boundary problem (1) is "very" discontinuous at $t=0$. We define it in the generalized sense as an element of the space $L^{2}\left(0, T ; L^{2}(0,1)\right)$ satisfying the following identity for all $\varphi \in H_{0}^{2,1}\left(Q_{T}\right)$ :

$$
\begin{gathered}
\int_{0}^{1} u(x, t) \varphi(x, t) \mathrm{d} x-\int_{0}^{T} \int_{0}^{1} u(x, t) \varphi_{t}(x, t) \mathrm{d} x \mathrm{~d} t \\
=\int_{0}^{T} \int_{0}^{1} u(x, t) \varphi_{x x} \mathrm{~d} x \mathrm{~d} t+\int_{0}^{T} \varphi\left(x^{0}, t\right) \mathrm{d} t,
\end{gathered}
$$

where

$$
\begin{aligned}
& H_{0}^{2,1}\left(Q_{T}\right) \\
& \quad=\left\{\varphi\left|\varphi, \varphi_{x}, \varphi_{x x}, \varphi_{t} \in L^{2}\left(Q_{T}\right), \varphi\right|_{x=0,1}=0\right\} .
\end{aligned}
$$

It is readily seen that

$$
u(x, t)=\sum_{i=1}^{\infty} 2 e^{-(\pi i)^{2} t} \sin \pi i x^{0} \sin \pi i x
$$

and

$$
u \in L^{2}\left(0, T ; L^{2}(0,1)\right) \cap C^{\infty}([0,1] \times(0, T]) .
$$

The linearity of (1) and (2) yields that this solution is unique.

2.2. Stationary point sensor. Suppose that we need to find the point $x^{0}$, based solely on the measurement data obtained from a sensor located at a given point $r \in(0,1)$ (see also Remark 2 below). In other words, we assume that we know the values of the function

$$
y(t)=u(r, t), \quad t \in[0, T] .
$$

Note that (4) implies that $u(r, \cdot) \in L^{1}(0, T) \cap C(0, T]$ for any $r, x^{0} \in(0,1)$ and, given the sensor's position $r \in$ $(0,1), u(r, \cdot) \in L^{2}(0, T)$ for a.a. $x^{0} \in(0,1)$ (and viceversa).

Given the location $r$ for a sensor and data $y(t), t \in$ $(0, T)$, a solution to this source localization problem can, in general, be set valued, in which case we cannot pinpoint the exact position of the unknown source. The way out here we see in a very careful choice of the site for our sensor which we link to the following problem.

Identifiability/sensor placement problem: Find a location $r$ for a sensor in (5) which can guarantee the uniqueness of the solution to the source localization problem at hand, regardless of any possible realization of measurement data. (Remember that we "do not know" $y(\cdot)$ in advance.)

2.3. Method of test-functions. Our further plan is to try to solve the source localization problem first. While doing it, we will try to distinguish conditions on the site $r$ for a sensor which solves the sensor placement problem.

Combining (3) and (5) provides us with the following functional equation in $x^{0}$ :

$$
\sum_{i=1}^{\infty} e^{-(\pi i)^{2} t} 2 \sin \pi i x^{0} \sin \pi i r=y(t), \quad t \in(0, T),
$$

which in turn is equivalent to the infinite system of algebraic equations, namely,

$$
\begin{aligned}
& \int_{0}^{T} \lambda(t)\left(\sum_{i=1}^{\infty} e^{-(\pi i)^{2} t} 2 \sin \pi i x^{0} \sin \pi i r \mathrm{~d} t\right) \\
&=\int_{0}^{T} \lambda(t) y(t) \mathrm{d} t, \quad \lambda \in L^{2}(0, T),
\end{aligned}
$$

where, given $r$, the integration is well defined for a.a. $x^{0} \in$ $(0,1)$ (see also 9 below)

In fact, to make (7) equivalent to (6), it is sufficient to consider $\lambda$ 's only from $L^{\infty}(0,1)$, in which case the integration in (7) is always well defined. However, we prefer to deal with a "simpler" Hilbert space $L^{2}(0,1)$ below.

Given any sensor's position $r \in(0,1)$, for any $\lambda \in$ $L^{2}(0, T)$ write

$$
\gamma_{\lambda}(x)=\sum_{i=1}^{\infty} 2\left(\int_{0}^{T} \lambda(t) e^{-(\pi i)^{2} t} \sin \pi i r \mathrm{~d} t\right) \sin \pi i x .
$$

Note that $\gamma_{\lambda} \in L^{2}(0,1)$, and (7) becomes

$$
\gamma_{\lambda}\left(x_{0}\right)=\int_{0}^{T} \lambda(t) y(t) \mathrm{d} t, \quad \forall \lambda \in L^{2}(0, T),
$$


where, as in (7), the left-hand side and the right-hand side are equal as two elements from $L^{2}(0,1)$ with respect to $x^{0} \in(0,1)$.

Denote by $\Gamma$ the set of all possible functions $\left\{\gamma_{\lambda} \mid\right.$ $\left.\lambda \in L^{2}(0, T)\right\}$. We will further refer to them as the testfunctions.

Remark 1. In (1) we assumed that the intensity of the source is one unit. Suppose now that it is any (positive) number $\nu$. Then in place of (9) we will have

$$
\nu \gamma_{\lambda}\left(x_{0}\right)=\nu \int_{0}^{T} \lambda(t) y(t) \mathrm{d} t, \quad \forall \lambda \in L^{2}(0, T),
$$

where $y(\cdot)$ is from (9). Cancelling $\nu$ gives us (9). Thus, the magnitude of intensity does not affect the process of finding $x^{0}$.

Assume now that it is possible to select $\lambda(t)$ so that the corresponding function $\gamma_{\lambda}$ is continuous and monoto$n e$ in $x$ on some interval $(\alpha, \beta) \subset(0,1)$. Then, if our source localization problem has a solution $x^{0}$ in $(\alpha, \beta)$, it will be the only point

$$
x^{0}=\gamma_{\lambda}^{-1}\left(\int_{0}^{T} \lambda(t) y(t) \mathrm{d} t\right) \in(\alpha, \beta) .
$$

Furthermore, if we manage to find two functions $\lambda_{1}$ and $\lambda_{2}$ such that the corresponding functions $\gamma_{\lambda_{1}}$ and $\gamma_{\lambda_{2}}$ are continuous and strictly monotone on, respectively, $(0, b)$ and $(c, 1)$, where $b>c$, then our localization problem has at most two solutions,

$$
x_{1}^{0}=\gamma_{\lambda_{1}}^{-1}\left(\int_{0}^{T} \lambda_{1}(t) y(t) \mathrm{d} t\right) \in(0, b)
$$

and

$$
x_{2}^{0}=\gamma_{\lambda_{2}}^{-1}\left(\int_{0}^{T} \lambda_{2}(t) y(t) \mathrm{d} t\right) \in(c, 1) .
$$

Now, if we can find the third function $\lambda_{3}$ such that the corresponding functions $\gamma_{\lambda_{3}}$ is continuous and strictly monotone on $\left[x_{1}^{0}, x_{2}^{0}\right]$, then our source localization problem will not have more than one solution in $(0,1)$.

Let us discuss now the role of the site $a$ for the sensor in the above argument and which of the associated calculations can be made "off-line."

- Provided that the given site $a$ for the sensor permits that, the functions $\lambda_{i}, \gamma_{\lambda_{i}}, i=1,2$, can be "prepared" in advance ("off line"). They do not require the knowledge of the data $y(t), t \in(0, T)$.
- On the contrary, in order to construct $\gamma_{\lambda_{3}}$, one needs to know the exact locations of $x_{1}^{0}$ and $x_{2}^{0}$ in $(0,1)$, which can only be found by using the "actual" measurement data. Thus, the latter requires "on-line" calculations.

- Moreover, we need such sensor placement that will allow us to find a suitable function $\gamma_{\lambda_{3}}$ regardless of the unknown, in advance, realization of the actual measurement data. (Otherwise, it will be "too late.")

Hence, if we want to be able to solve the source localization problem at hand uniquely following the above argument, we should place our sensor at such a place that the corresponding set $\Gamma$ is "sufficiently rich," namely,

- $\Gamma$ should contain at least two functions $\gamma_{\lambda_{i}}, i=1,2$, that are continuous and strictly monotone, respectively, on some intervals $(0, b)$ and $(c, 1), 0<c<$ $b<1$.

- It should also contain infinitely many functions $\gamma_{\lambda_{(k)}}, k=1, \ldots$, which are continuous and strictly monotone, respectively, on some sequence of intervals $\left(c_{k}, b_{k}\right)$, where $c_{k} \rightarrow 0+$ and $b_{k} \rightarrow 1-$ as $k \rightarrow \infty$.

As a matter of fact, the second of the above conditions is sufficient to solve the source localization problem uniquely. Indeed, if there are two possible distinct solutions $x_{1}^{0}$ and $x_{2}^{0}$ in $(0,1)$, then they have to lie in one of the intervals $\left(c_{k}, b_{k}\right)$, which contradicts the fact that the equation

$$
\gamma_{\lambda_{(k)}}\left(x^{0}\right)=\int_{0}^{T} \lambda_{(k)}(t) y(t) \mathrm{d} t
$$

admits only a unique solution $x^{0}$ in $\left(c_{k}, b_{k}\right)$.

The question of the richness of the set $\Gamma$ leads us to the so-called controllability problem, which plays one of the central roles in mathematical control theory.

\subsection{Source identifiability and the dual controllability} problem. Let us note that the set of all $\gamma_{\lambda}(x)$ is the set of all the states of the following boundary problem at time $T$ :

$$
\begin{gathered}
z_{t}=z_{x x}+\lambda(T-t) \delta(x-r) \quad \text { in }(0,1) \times(0, T)=Q_{T}, \\
\left.z\right|_{x=0,1}=0, \quad z(x, 0)=0,
\end{gathered}
$$

that is,

$$
\Gamma=\left\{z(x, T)=\gamma_{\lambda}(x) \mid \lambda \in L^{2}(0, T)\right\} \subset L^{2}(0,1) .
$$

Indeed, it was shown, e.g., by Ladyzhenskaja et al. (1968) that solutions to (13) (understood in the 
generalized sense analogous to (2) lie in the space $C\left([0, T] ; L^{2}(0,1)\right) \cap H_{0}^{1,0}\left(Q_{T}\right)$, where $H_{0}^{1,0}\left(Q_{T}\right)=\{\varphi \mid$ $\left.\varphi, \varphi_{x} \in L^{2}\left(Q_{T}\right),\left.\varphi\right|_{x=0,1}=0\right\}$. Then the method of separation of variables yields that $z(x, T)$ has the representation (8). We stress, in particular, that some of the testfunctions $\gamma_{\lambda}$ can be discontinuous.

The following definition, classical in control theory, (Dolecki and Russell, 1977), describes the "richness" of the set $\Gamma$.

Definition 1. The system (1) is approximately controllable in a space $H$ at time $T$ if the set of all its states at time $t=T$, that is, the set $\Gamma$ is dense in $H$.

We have the following approximate controllability result in $H=H^{2}(0,1) \cap H_{0}^{1}(0,1)$ (see, e.g., the work of Khapalov (1994) where

$$
\begin{aligned}
& H_{0}^{1}(0,1)=\left\{\varphi\left|\varphi, \varphi_{x}, \in L^{2}\left(Q_{T}\right), \varphi\right|_{x=0,1}=0\right\}, \\
& H^{2}(0,1)=\left\{\varphi \mid \varphi, \varphi_{x}, \varphi_{x x} \in L^{2}\left(Q_{T}\right\} .\right.
\end{aligned}
$$

Proposition 1. Let the sensor position $r$ be any irrational number from $(0,1)$. Then, for any $T>0$, the set $\Gamma$ is dense in the space $H^{2}(0,1) \cap H_{0}^{1}(0,1)$.

The proof is based on the fact (see, e.g., the work of Fattorini and Russell (1974) that $\left\{e^{-(\pi i)^{2} t}, i=1, \ldots\right\}$ form a Riesz basis in $L^{2}(0, T)$. In other words, for any $T>0$, there is a (biorthogonal) sequence $\left\{q_{i}(t)\right\}_{i=0}^{\infty}$ such that

$$
\int_{0}^{T} e^{-(\pi i)^{2} t} q_{k}(t) \mathrm{d} t=\left\{\begin{array}{lll}
1 & \text { if } & i=k \\
0 & \text { if } & i \neq k
\end{array}\right.
$$

This implies, in particular, that $\Gamma$ contains all the functions represented by finite sums like

$$
\sum_{k=1}^{K} \alpha_{k} \sin \pi k x, \quad K=1, \ldots,
$$

which are dense in $H^{2}(0,1) \cap H_{0}^{1}(0,1)$.

Now we are in a position to prove the following identifiability result.

Theorem 1. Let the sensor position $r$ be any irrational number from $(0,1)$. Then, for any $T>0$ and any realization of measurement data $y(t), t \in(0, T)$, the source localization problem admits a unique solution only.

Proof. Consider any interval $[0, b] \subset[0,1)$ and any continuously differentiable function $g$ on $[0,1]$ which vanishes at $x=0,1$ and whose derivative on $[0, b]$ is equal to 1 . It follows from Proposition 1 that this function can be approximated by functions from $\Gamma$ arbitrarily closely in the norm of the space $C^{1}[0,1]$. Hence, we can find a function in $\Gamma$ which has the form (14) and whose derivative is strictly positive on $(0, b)$, and select it as $\gamma_{\lambda_{1}}$ in the argument of Subsection 2.3. Analogously, we can construct functions $\gamma_{\lambda_{2}}$ and $\gamma_{\lambda_{(k)}}, k=1, \ldots$, which will allow us to solve the localization problem at hand uniquely. This completes the proof of Theorem 1 .

2.5. Discussion of 1-D results and further plans. An immediate drawback of Proposition 1 and Theorem 1 is that one has to place a point sensor at an "irrational" site. Clearly, this creates a serious problem in applications. We emphasize that this difficulty is not due to our method of test-functions but is an intrinsic property of this physical phenomenon (that is, as described by the model (1)). Indeed, if the sensor is placed at a "rational" point $r$, then the set of test functions $\Gamma$ does not have in their representation (8) infinitely many terms, namely, those that contain the functions $\sin \pi k x$ vanishing at $r$. In other words, such a sensor is unable to "detect" the contribution of these terms.

On the other hand, for any given sensor site $r$ and any $\lambda \in L^{2}(0, T)$, the corresponding function $\gamma_{\lambda}$ can be used to uniquely identify a possible location of an unknown source $x^{0}$ within any subinterval of $(0,1)$, where it is strictly monotone and continuous, see (11)-12). This opens a good perspective for "numerical experiments" in applications.

Remark 2. (Finitely many point sensors.) Let us note that, if, instead of one stationary point sensor, we take any finite number of them, it does not principally change the situation here. Indeed, if all the sensor are at "rational" sites, say, $\left\{p_{1} / q_{1}, \ldots, p_{N} / q_{N}\right\}$, then in the expression (8) all terms which are multiple with the number $q_{1} \cdot \ldots \cdot q_{N}$ vanish.

Example 1. One practical problem. A practical situation is the case when all the "potential" locations of an unknown source are known in advance. They can, e.g., be the sites where the canisters filled with waste are stored in a pool or underground, which at some moment of time can start "leaking." This type of storage is supposed to be monitored by a reasonably secure system of sensors in order to timely reveal possible contamination.

For example, suppose that a potential source can emerge only within one of the given $N$ subintervals $\Omega_{i}, i=1, \ldots, N$ of the space domain $(0,1)$. Then, to make a conclusion in what subinterval $\Omega_{i}$ the unknown source actually lies, it is sufficient to find $N$ test-functions, each of which is "principally distinct" from others only on one of the above-mentioned intervals.

We may choose, e.g.,

$$
\gamma_{\lambda_{i}}(x)= \begin{cases}1, & x \in \Omega_{i}, \\ 0, & x \in(0,1) \backslash \Omega_{i} .\end{cases}
$$


Then, if the source emerged, say, in $\Omega_{i *}$, then (as Eqns. (11)-12) imply)

$$
\int_{0}^{T} \lambda_{i}(x) y(t) \mathrm{d} t= \begin{cases}1, & \text { if } i=i_{*}, \\ 0, & \text { if } i \neq i_{*} .\end{cases}
$$

Note that the corresponding $\lambda_{i}$ 's can be found "off-line."

\section{General 1-D model with mobile point sensors}

Let us consider now the following, rather general, boundary problem:

$$
\begin{array}{rlrl}
u_{t}=u_{x x}+b(x, t) u_{x}+a(x, t) u & \text { in } Q_{T}, \\
\left.u\right|_{x=0,1}=0, & \left.u\right|_{t=0} & =u_{0}, \\
a & \in C\left(\bar{Q}_{T}\right), & b & \in C^{0,1}\left(\bar{Q}_{T}\right) .
\end{array}
$$

Assume first that (as in Section 2) this process is excited by a pointwise source $u_{0}(x)=\delta\left(x-x^{0}\right)$, whose location is to be found, based on data obtained from a stationary pointwise sensor located at the given site $r$.

We immediately see that those techniques of the previous section that are based on the Fourier series approach do not apply to 16 .

- Firstly, the formula (6) makes use of the explicit Fourier series representation of solutions to (1), while the concept of eigenvalues and eigenfunctions does not apply to the time-varying system (16).

- Secondly, due to the same reason, the arguments of Proposition 1 and Theorem 1, involving the series of exponential functions, do not apply either.

Nonetheless, we intend to show that the method of the testfunction is still perfectly applicable to the system (16) in the form of Eqn. (9) and the formula (14), which link the solution of the source localization problem to the controllability property of the dual control problem.

Also, to cope with the difficulties arising in addition due to a more general time-varying nature of (16), we will make two changes in the models for sensors and sources.

Two mobile sensors. We now assume that we have two point sensors that can move along the given trajectories $x=s_{l}(t), t \in(0, T), l=1,2$ and the measurement data are respectively represented by the functions

$$
\begin{aligned}
y(t)=\left(y_{1}(t), y_{2}(t)\right), \quad y_{l}(t)= & u\left(s_{l}(t), t\right), \\
t & \in[0, T], \quad l=1,2 .
\end{aligned}
$$

Note that this two-sensor model includes, of course, our previous one-sensor model as a particular case (to see that, assume, e.g., that $\left.s_{1}(t) \equiv 0\right)$. However, we intend to show below that the use of two mobile sensors may provide us with somewhat more versatile results (though not principally different).

"Zone" model for a source. Alternatively we can consider the case when the unknown source is modeled as

$$
\begin{aligned}
u_{0}(x)= & u_{0}\left(x, x^{0}\right) \\
= & \frac{1}{\operatorname{meas}\left\{S_{h}\left(x^{0}\right) \cap(0,1)\right\}} \chi_{S_{h}\left(x^{0}\right)} \\
= & \frac{1}{\operatorname{meas}\left\{S_{h}\left(x^{0}\right) \cap(0,1)\right\}} \\
& \cdot \begin{cases}1 & \text { if } x \in S_{h}\left(x^{0}\right) \cap(0,1), \\
0 & \text { if } x \in(0,1) \backslash S_{h}\left(x^{0}\right),\end{cases}
\end{aligned}
$$

where $x^{0}$ is to be found and $S_{h}\left(x^{0}\right)=\left(x^{0}-h, x^{0}+h\right)$ is the "effective" zone of the source of a given radius $h \in$ $(0,1)$. We assume that the intensity of the source does not change over $(0, T)$ and is equal to one unit (see Remark 1). This type of sources will be considered in Section 6 .

Dual control model. Introduce the controlled boundary problem dual of (16) as follows:

$$
\begin{gathered}
z_{t}=z_{x x}-(b(x, T-t) z)_{x}+a(x, T-t) z \\
+\lambda_{1}(T-t) \delta\left(x-s_{1}(T-t)\right) \\
+\lambda_{2}(T-t) \delta\left(x-s_{2}(T-t)\right) \quad \text { in } Q_{T} \\
\left.z\right|_{x=0,1}=0,\left.\quad z\right|_{t=0}=0 .
\end{gathered}
$$

Following the terminology of control theory, we regard $\lambda_{l}(T-t), t \in(0, T), l=1,2$ in (18) as "controls".

Multiplying (16) with any $u_{0}$ in $L^{2}(0,1)$ by $z(x, T-$ $t)$ and further integrating by parts over $Q_{T}$ yields the classical duality relation:

$$
\begin{aligned}
& \int_{0}^{1} z(x, T) u_{0}(x) \mathrm{d} x \\
& \quad=\int_{0}^{T} \lambda_{1}(t) u\left(s_{1}(t), t\right) \mathrm{d} t+\int_{0}^{T} \lambda_{2}(t) u\left(s_{2}(t), t\right) \mathrm{d} t, \\
& \forall \lambda \in L^{2}(0, T) .
\end{aligned}
$$

Test-functions. Now, for $u_{0}(\cdot)=u_{0}\left(\cdot, x^{0}\right)$ as in (17) we introduce the test-functions following (14):

$$
\left.\gamma_{\lambda}(x)=z(x, T), \quad \lambda=\left(\lambda_{1}, \lambda_{2}\right) \in\left[L^{2}(0, T)\right]^{2}\right),
$$

where $\lambda=\left(\lambda_{1}, \lambda_{2}\right)$ is the pair of controls "producing" $z(\cdot, T)$ as the state of the system 18$)$ at time $T$. Respectively, we will use the following version of Eqn. (19) (in 
place of (9)) to find $x^{0}$ :

$$
\begin{aligned}
& \int_{0}^{1} \gamma_{\lambda}(x) u_{0}\left(x, x^{0}\right) \mathrm{d} x \\
& \quad=\int_{0}^{T} \lambda_{1}(t) y_{1}(t) \mathrm{d} t+\int_{0}^{T} \lambda_{2}(t) y_{2}(t) \mathrm{d} t, \\
& \forall \lambda_{l} \in L^{2}(0, T), \quad l=1,2 .
\end{aligned}
$$

Throughout this paper we assume the following conditions on the curves $s_{l}, l=1,2$, which are to ensure both the mathematical well-posedness of the system at hand and to preserve their physical meaning as the trajectories for the point sensors.

\section{Assumption 1.}

- We assume that the functions $s_{1}$ and $s_{2}$ are continuous on $[0, T]$ with values in $[0,1]$, although these functions may be defined/introduced only on some subsegment(s) of $[0, T]$. (The latter means that the associated sensors "act" only where $s_{i}$ are defined and are "inactive" at other times.)

- We also assume that any horizontal line $\{(x, t) \mid t=$ $\left.t_{*}\right\}$ can cross either of the trajectories $s_{i}$ at most at one point. (Indeed, if $s_{i}, i=1,2$, represent the paths of point sensors, then at every moment of time they can be supported only at single points.)

It is known that the systems (16) and (18) (see, e.g., the results of Ladyzhenskaja et al. (1968) and Khapalov (2001)) possess unique solutions in the Banach space $\mathcal{B}=$ $C\left([0, T] ; L^{2}(0,1)\right) \cap H_{0}^{1,0}\left(Q_{T}\right)$. In the work of Khapalov (2001) it was also shown that the traces of solutions to (16) on the curves $s_{l}(\cdot), l=1,2$ are well defined as elements of $L^{2}(0, T)$.

We have the following identifiability result.

Theorem 2. Let the set $\Gamma$ of all test-functions (20) be dense in $L^{2}(0,1)$ or, which is the same, the system (18) be approximately controllable in $L^{2}(0,1)$ at time $T$.

- Then for every possible realization of data $y(t), t \in$ $(0, T)$ the source localization problem for the system (16) admits only a unique solution in $(0,1)$.

- For an arbitrary $\varepsilon>0$ there exists a test-function $\gamma_{\lambda_{\varepsilon}}$ such that for any realization of data $y(t), t \in$ $(0, T)$ all possible solutions $x^{0}$ from $(0,1)$ to Eqn. (21) with $\gamma_{\lambda_{\varepsilon}}$ in place of $\gamma_{\lambda}$, including, in particular, the actual location of the unknown source, lie within an interval whose length does not exceed $\varepsilon+h^{2}$.

We regard the second of the above results as the $\varepsilon h$-identifiability of $x^{0}$. It states that if we can "afford" an error of "size" $\varepsilon+h^{2}$, then, under the assumptions of Theorem 2, the infinite system of equations (21) can be reduced to just one equation with a carefully chosen testfunction.

Proof. In fact, uniqueness follows from the observability property, which is tantamount to the approximate controllability assumption of Theorem 2, see Definition 2 and Remark 3 in the next section.

Fix any $\delta>0$. Since $\Gamma$ is dense in $L^{2}(0,1)$, we can select $\lambda$ so that

$$
\gamma_{\lambda}(x)=x+g(x)
$$

where

$$
\left(\int_{0}^{1} g^{2}(x) \mathrm{d} x\right)^{1 / 2} \leq \delta .
$$

Then, owing to (17),

$$
\begin{aligned}
& \int_{0}^{1} \gamma_{\lambda}(x) u_{0}\left(x, x^{0}\right) \mathrm{d} x \\
& =\int_{0}^{1}(x+g(x)) u_{0}\left(x, x^{0}\right) \mathrm{d} x \\
& =\frac{1}{\operatorname{meas}\left\{S_{h}\left(x^{0}\right) \cap(0,1)\right\}} x^{0} \\
& +\frac{1}{\operatorname{meas}\left\{S_{h}\left(x^{0}\right) \cap(0,1)\right\}} \underset{\left\{\tau \mid \tau+x^{0} \in S_{h}\left(x^{0}\right) \cap(0,1)\right\}}{\int} \int_{\left\{S_{h}\left(x^{0}\right) \cap(0,1)\right\}} g(x) \mathrm{d} x,
\end{aligned}
$$

where

$$
\text { meas }\left\{S_{h}\left(x^{0}\right) \cap(0,1)\right\} \in(h, 2 h] \text { for } \quad x^{0} \in(0,1) .
$$

Hence

$$
\begin{aligned}
& \int_{0}^{1} \gamma_{\lambda}(x) u_{0}\left(x, x^{0}\right) \mathrm{d} x \\
& \quad=\frac{1}{\operatorname{meas}\left\{S_{h}\left(x^{0}\right) \cap(0,1)\right\}} x^{0}+\rho\left(x^{0}, h\right),
\end{aligned}
$$

where

$$
\left|\rho\left(x^{0}, h\right)\right| \leq \frac{h}{4}+\frac{\delta}{\sqrt{2 h}} .
$$

Respectively, 21 yields

$$
\begin{aligned}
x^{0}= & \operatorname{meas}\left\{S_{h}\left(x^{0}\right) \cap(0,1)\right\} \\
& \times\left(\sum_{l=1}^{2} \int_{0}^{T} \lambda_{l}(t) y_{l}(t) \mathrm{d} t-\rho\left(x^{0}, h\right)\right) .
\end{aligned}
$$


Hence

$$
c_{1} \leq x^{0} \leq c_{2}
$$

where

$$
\begin{aligned}
c_{1}= & \operatorname{meas}\left\{S_{h}\left(x^{0}\right) \cap(0,1)\right\} \sum_{l=1}^{2} \int_{0}^{T} \lambda_{l}(t) y_{l}(t) \mathrm{d} t \\
& -\frac{h^{2}}{2}-\delta \sqrt{2 h}, \\
c_{2}= & \operatorname{meas}\left\{S_{h}\left(x^{0}\right) \cap(0,1)\right\} \sum_{l=1}^{2} \int_{0}^{T} \lambda_{l}(t) y_{l}(t) \mathrm{d} t \\
& +\frac{h^{2}}{2}+\delta \sqrt{2 h} .
\end{aligned}
$$

This provides the conclusion of Theorem 2 with any $\delta<$ $\varepsilon / 2 \sqrt{2 h}$.

\section{Controllability and observability}

In Theorem 2 the crucial technical condition is the approximate controllability of (18) at time $T>0$ in the phasespace $L^{2}(0,1)$, which is linked to the "right" choice of the trajectories for mobile sensors. In turn, it is well known (Dolecki and Russell, 1977) that the approximate controllability of (18) is tantamount to the so-called observability property of 16 at time $T>0$ with $u_{0} \in L^{2}(0,1)$.

Definition 2. The system with $u_{0} \in L^{2}(0,1)$ is said to be observable at time $T>0$ if the mapping

$$
\text { Measurement Data } \rightarrow \text { State }
$$

is one-to-one. In other words, the following implication holds:

$$
\begin{aligned}
u\left(s_{l}(t), t\right)=0 \quad t \in(0, T), & l=1,2 \\
& \Longrightarrow u \equiv 0 \text { in } Q_{T} .
\end{aligned}
$$

Remark 3. Note that 24), in particular, implies the unique identification of the source function (17) as an element of $L^{2}(0,1)$ as well. In this respect, we would like to emphasize that Theorem 2 in the form of the formula 23 provides us also with a tool for calculating $x^{0}$.

The equivalence of the approximate controllability of (18) in $L^{2}(0,1)$ at time $T$ and the observability of (16) with $u_{0} \in L^{2}(0,1)$ at time $T$ follows from the duality relation (19).

Indeed, if, for example, the linear manifold of all $z(\cdot, T)$ is not dense in $L^{2}(0,1)$, then there is a $u_{0} \neq 0$ such that

$$
\int_{0}^{1} z(x, T) u_{0}(x) \mathrm{d} x=0, \quad \forall \lambda_{l} \in L^{2}(0, T), \quad l=1,2 .
$$

Owing to (19), this yields $u\left(s_{l}(\cdot), \cdot\right) \equiv 0, \quad l=1,2$, which implies that $u_{0}=0$, whenever (16) is observable (and vice-versa).

Thus, to establish controllability (and, in particular, source identifiability), it is sufficient to establish the observability of (16) with $u_{0} \in L^{2}(0,1)$.

We now intend to show that for any non-degenerate $\omega=\left(l_{1}, l_{2}\right) \subseteq(0,1)$ one can select two curves $s_{1}$ and $s_{2}$ lying in $\omega \times(0, T)$ which ensure (24). Our results are linked to the geometric Assumptions 2 and 3 on these curves given below.

We start with the following.

Assumption 2. ("The same starting point.") There is an interval $\left[t_{1}, t_{2}\right] \subset(0, T]$ such that the functions $x=$ $s_{1}(t)$ and $x=s_{2}(t), \quad t \in\left[t_{1}, t_{2}\right]$ are continuous and one-to-one on it with values in $[0,1]$ and

$$
s_{1}\left(t_{1}\right)=s_{2}\left(t_{1}\right), \quad s_{1}(t)<s_{2}(t), \quad \forall t \in\left(t_{1}, t_{2}\right] .
$$

This condition means that the curves $s_{1}$ and $s_{2}$ have a common point at time $t_{1}$.

We start with the following immediate result.

Theorem 3. Let $T>0$ be given, Assumptions 1 and 2 hold, and $a(x, t) \leq 0$ in the horizontal strip $\mathcal{A}(\cdot)=$ $\left\{(x, t) \mid x \in[0,1], t \in\left[t_{1}, t_{2}\right]\right\}$. Then the system (16), (17) is ch-identifiablle.

Proof. It is sufficient to show that (16) is observable in the sense of (24). Indeed, let solution $u$ to (16) with $u_{0} \in$ $L^{2}(0,1)$ vanish on the curves $s_{1}(t)$ and $s_{2}(t), t \in\left[t_{1}, t_{2}\right]$ "emitted" from the point $\left(s_{1}\left(t_{1}\right)=s_{2}\left(t_{1}\right), t_{1}\right)$. Then, due to the maximum principle (Friedman, 1964), $u$ vanishes everywhere in the set

$$
\mathcal{A}_{1}(\cdot)=\left\{(x, t) \mid s_{1}(t) \leq x \leq s_{2}(t), t \in\left[t_{1}, t_{2}\right]\right\} .
$$

Hence, by the unique continuation property (Saut and Scheurer, 1987), $u$ vanishes in the horizontal layer $\mathcal{A}(\cdot)$. By backward (and forward) uniqueness, this solution vanishes in $Q_{T}$. Thus, we have (24) and hence the approximate controllability of dual [18), which allows us to apply Theorem 2.

Remark 4. The maximum principle we used in the above was shown by Friedman (1964) for the classical solutions to (16). Due to the smoothing effect, it can be applied directly to (16) on any $\left[t_{1}, t_{2}\right], \quad 0<t_{1}<t_{2} \leq T$ when $a=b=0$, i.e., when (16) is the standard heat equation. We can extend this result to the general case described in Theorem 3 dealing with (continuous in $\mathcal{A}(\cdot)$ ) solutions to (16) by the density argument (see also the results of Khapalov (2001)).

The case of a single sensor. Due to the zero boundary condition in (16), any solution $u$ vanishes on the lines 
$x=0$ and $x=1$. Hence, if we assume that we have an "imaginary" stationary sensor located on either of the boundaries of $(0,1)$, it will always provide us with the zero "measurement data." Therefore, in the argument of Theorem 3 we can assume, for example, that trajectory $s_{1}$ is the boundary $\{(x, t) \mid x=0, t \in(0, T)\}$. Then, the argument of Theorem 3 will work just for any single sensor with a trajectory $s_{2}(t), t \in(0, T)$ such that $s_{2}\left(t_{1}\right)=0$ and $s_{2}(t)$ is strictly monotone on some $\left(t_{1}, t_{2}\right) \subset(0, T]$. In other words, the sensor should move during some time interval from the boundary of the space interval towards its interior.

Let us consider now the general case of (16), that is, not assuming that $a(x, t) \leq 0$ as in Theorem 3 .

\section{Assumption 3.}

- In addition to Assumptions 1 and 2, assume that on the interval $\left[t_{1}, t_{2}\right] \subset[0, T]$ (that is, $t_{1}$ can also be the initial moment) the functions $s_{1}$ and $s_{2}$ are respectively strictly monotone decreasing and increasing.

- Assume that the connected (by Assumption 2) geometric curve $s$ in $\bar{Q}_{T} \subset R^{2}$, composed of the curves $s_{1}$ and $s_{2}$ on the interval $\left[t_{1}, t_{2}\right]$, admits the following representation:

$$
s(\cdot)=\{(x, t) \mid(x, t) \in \mathcal{A}(\cdot), F(x, t)=0\},
$$

where $F$ is an element of $C^{2,1}(\mathcal{A}(\cdot))$ and

$$
\begin{gathered}
F_{t}(x, t)-b_{x}(x, t) F(x, t)-b(x, t) F_{x}(x, t) \\
+F_{x x}(x, t) \leq 0, \quad \forall(x, t) \in \mathcal{A}_{1}(\cdot), \\
F(x, t)>0 \forall(x, t) \in \operatorname{int}\left\{\mathcal{A}_{1}(\cdot)\right\}, \\
F(x, t) \leq 0 \forall(x, t) \in \mathcal{A}_{2}(\cdot)=\mathcal{A}(\cdot) \backslash \mathcal{A}_{1}(\cdot) .
\end{gathered}
$$

Our main result here is based on the following lemma (proven in detail by Khapalov (2001)).

Lemma 1. Let $T>0$ be given and Assumptions 1-3 hold. Then for any solution to the system (16) the following observability estimate holds:

$$
\begin{aligned}
& \|u(\cdot, T)\|_{L^{2}(0,1)} \\
& \leq C\left(\int_{t_{1}}^{t_{2}}\left(u^{2}\left(s_{1}(t), t\right)+u^{2}\left(s_{2}(t), t\right)\right) \mathrm{d} t\right)^{1 / 2},
\end{aligned}
$$

where $C$ is some positive constant.

Note that 27) implies the uniqueness property 24) due to the backward uniqueness of solutions to (16). Its direct consequence is the approximate controllability of (18) in $L^{2}(\Omega)$ at time $T$, which, being combined with Theorem 2 , yields the following result.
Theorem 4. Let $T>0$ be given and Assumptions 1-3 hold. Then the system (16), (17) is Eh-identifiable.

Both Lemma 1 and Theorem 4 can be extended to the case of a single sensor as discussed after Theorem 3.

Example 2. Convection-diffusion equation and a sensor moving at constant speed. Let (16) have the form

$$
\begin{gathered}
u_{t}=u_{x x}+b u_{x} \text { in } Q_{T}, \\
\left.u\right|_{x=0,1}=0,\left.\quad u\right|_{t=0}=u_{0},
\end{gathered}
$$

where the velocity of convection $b$ is assumed to be constant. For this model let us consider a sensor that moves also at a constant speed $\alpha>0$ across $(0,1)$ from the point $x=0$ to the point $x=\alpha\left(T-t_{1}\right)$ during the time-interval $\left(t_{1}, T\right), 0<t_{1}<T$.

Theorem 3 immediately yields that 28 is $\varepsilon h$ identifiable, regardless of the value of $b$. However, to apply Theorem 3, we need to assume that $t_{1}>0$, i.e., that the sensor initiates its motion after the process has begun. (Recall that we need the continuity of solutions to (28) on $\left[t_{1}, T\right]$ in Theorem 3.)

Let us now compare this result with Lemma 1 and Theorem 4 , which in addition to $\varepsilon h$-identifiability provide the estimate (27) ensuring the well-posedness of the identification process with respect to measurement data.

Note that in Theorem 2 we can also choose $t_{1}=0$, in which case the above-mentioned sensor trajectory will be described by the equation

$$
F(x, t)=-x+\alpha t=0
$$

and

$$
\mathcal{A}_{1}(\cdot)=\left\{(x, t) \in \bar{Q}_{T} \mid x \leq \alpha t\right\} .
$$

If $0<T<1 / \alpha$, then our sensor moves between the points $x=0$ and $x=\alpha T<1$. For $\alpha>0$, the condition (25) gives

$$
0<\alpha \leq-b .
$$

Hence, to make Theorem 4 and the estimate 27) work, we need to assume that the velocity of convection is negative and its magnitude is greater that the speed of the sensor.

Example 3. The case with no convection. In Example 1, we can also consider a parabolic curve like

$$
F(x, t)=-(x-0.5)^{2}+\alpha t=0,
$$

whose two branches, on the left and on the right of the line $x=0.5$, are assigned to be the trajectories for two point sensors:

$$
s_{1}(t)=0.5-\sqrt{\alpha t}, \quad s_{2}(t)=0.5+\sqrt{\alpha t}, \quad t \in[0, T] .
$$

To satisfy Theorem 4 , one may select, for example, $\alpha \in$ $(0,2], T<0.25 / \alpha$, while setting $b=0$, i.e., omitting convection. Note that our point controls move within the interval $[0.5-\sqrt{\alpha T}, 0.5+\sqrt{\alpha T}]$. (For instance, for $\alpha=$ $0.4, T=0.1$ this interval will be $[0.3,0.7]$.) 
Example 4. The general case. The parabolic trajectories as in Example 2 will satisfy any $a$ and $b$ described in Eqn. (16). Indeed, in this case, (26) holds as before, and (25) is as follows:

$$
\begin{aligned}
\alpha & -b(x, t)(-2(x-0.5)) \\
& -b_{x}(x, t)\left(-(x-0.5)^{2}+\alpha t\right)-2 \leq 0, \\
& -\sqrt{\alpha t} \leq x-0.5 \leq \sqrt{\alpha t}, \quad t \in\left(t_{1}, t_{2}\right) .
\end{aligned}
$$

It will hold if, e.g., $t_{1}=0$, while $\alpha \in(0,2)$ and $t_{2}$ is sufficiently small.

\section{Source acting in time}

In the above, we considered the case when the unknown source acted as a "one-time event" at the initial moment of time only. In this section we analyze the sources that are active continuously in time. They are relevant, in particular, to modeling "leaks" of hazardous materials.

Example 5. We refer, for example, to the problem of risk assessment of a radioactive waste repository in deep geological layers. It is associated with the transport of radionuclides by groundwater, see, e.g., the works of Dagan (1987; 1989), Devooght and Smidts (1996), PAGIS (1989), Williams (1992; 1993). In particular, the following model, described by the advective-dispersal equation in one or two-dimensional media (compare it with 16 and (34) below), was considered by Dagan (1987; 1989):

$$
\begin{aligned}
& \left(m R \frac{\partial}{\partial t}+\nabla(q(x, t) \cdot)-m D_{m}(x, t) \Delta\right. \\
& \left.-\nabla\left(D_{l} \nabla \cdot\right)+\lambda m R\right) \psi(x, t)=Q(t) \delta\left(x-x_{0}\right),
\end{aligned}
$$

where $\psi(x, t)$ constitutes the radionuclide concentration at position $x$ and time $t, Q(t)$ stands for the intensity of the source located at the position $x_{0}$ at time $t$, and $m, R, q, D_{m}, D_{l}$ are the parameters, which are functions of position and time. In particular, $D_{m}$ is the "molecular" diffusion coefficient due to a Brownian motion at the pore scale, $D_{l}$ is the local "mechanical" dispersion tensor, representing the effect of the groundwater flow passing through the tortuous structure of the geological medium and causing a dispersion of the concentration cloud. The retardation factor $R$ models the reversible exchange between the solution and the matrix, e.g., by adsorption and desorption; and $\lambda$ is the decay constant of the radionuclide. (Other examples relevant to the mathematical models analyzed in this paper can also be found, e.g., in the publications of Dagan $(1987 ; 1989)$, Devooght and Smidts (1996), PAGIS (1989), Williams (1992; 1993), see also the bibliography therein.)

Consider now the following very simple one dimensional model:

$$
u_{t}(x, t)=u_{x x}(x, t)+\delta\left(x-x^{0}\right), \quad(x, t) \in Q_{T},
$$

$$
\left.u\right|_{x=0,1}=0, \quad u(x, 0)=0,
$$

which describes the process generated by a source acting continuously-in-time at the unknown site $x^{0}$. We assume that the intensity of the source is constant during a ("small") time-interval $(0, T)$. As noticed in Remark 1, without loss of generality we may assume that it is equal to one unit.

Using the methods of Sections 2 and 3, we can obtain that the solution to (30) is an element of the space $L^{2}\left(0, T ; L^{2}(0,1)\right) \cap H_{0}^{1,0}\left(Q_{T}\right)$, represented by the following formula:

$$
\begin{aligned}
u(x, t) & =\sum_{k=1}^{\infty} 2 \int_{0}^{t} e^{-(\pi k)^{2}(t-\tau)} \sin \pi k x^{0} \mathrm{~d} \tau \sin \pi k x \\
& =2 \sum_{k=1}^{\infty} \frac{1-e^{-(\pi k)^{2} t}}{(\pi k)^{2}} \sin \pi k x^{0} \sin \pi k x .
\end{aligned}
$$

Suppose that we need to find the point $x^{0}$, based on the measurement data $y(t), t \in(0, T)$ obtained from a sensor located at a given point $r \in(0,1)$ as described in (5). Then, instead of Eqns. (8) and (9), we have

$$
\gamma_{\lambda}\left(x_{0}\right)=\int_{0}^{T} \lambda(t) y(t) \mathrm{d} t, \quad \forall \lambda \in L^{2}(0, T),
$$

where

$$
\begin{aligned}
\gamma_{\lambda}(x) & =\int_{0}^{T} \lambda(t) u_{\left(x^{0}\right)}(r, t) \mathrm{d} t \\
& =2 \sum_{k=1}^{\infty} \int_{0}^{T} \lambda(t) \frac{1-e^{-(\pi k)^{2} t}}{(\pi k)^{2}} \mathrm{~d} t \sin \pi k r \sin \pi k x,
\end{aligned}
$$

and $u_{\left(x^{0}\right)}(x, t)$ represents the solution to (30).

The following result is very similar to Theorem 1 .

Theorem 5. Let the position of sensor $r$ be any irrational number from $(0,1)$. Then, for any $T>0$ and any realization of measurement data $y(t), \quad t \in(0, T)$ the source localization problem for Eqn. (30) admits a unique solution only.

Indeed, based on the formulas (31)- 33), we can apply the argument of Proposition 1 almost identically, while using now the sequence $\left\{q_{k}^{*}, k=1, \ldots\right\}$ biorthogonal to the sequence of $\left\{1, e^{-\pi^{2} t}, \ldots, e^{-(\pi k)^{2} t}, \ldots\right\}$ in place of $\left\{e^{-\pi^{2} t}, \ldots, e^{-(\pi k)^{2} t}, \ldots\right\}$.

Theorem 5 have the same drawback as Proposition 1. Namely, its proof is heavily based on the explicit formula for solutions to (30) and involves the "irrational" sites for sensor placement. 
To get results more general than Theorem 5, one needs to study the set of test-functions given by $(33)$ in the fashion of Section 4.

\section{Multidimensional models}

Let $\Omega$ be a bounded, open, connected set in $\mathbb{R}^{n}$ with boundary $\partial \Omega$. In $\Omega$ we consider the following homogeneous Dirichlet problem for the parabolic equation:

$$
\begin{aligned}
\frac{\partial u}{\partial t} & =\mathcal{A}(x, t) u \\
= & \sum_{i, j=1}^{n} \frac{\partial}{\partial x_{i}}\left(a_{i j}(x, t) \frac{\partial u}{\partial x_{j}}\right)+\sum_{i=1}^{n} b_{i}(x, t) \frac{\partial u}{\partial x_{i}} \text { (34) } \\
& +a(x, t) u \quad \text { in } Q_{T}=\Omega \times(0, T), \\
\left.u\right|_{\Sigma_{T}} & =0 \text { in } \Sigma_{T}=\partial \Omega \times(0, T),\left.\quad u\right|_{t=0}=u_{0} \in L^{2}(\Omega) .
\end{aligned}
$$

We assume that for all $\xi_{i} \in \mathbb{R}$ a.e. in $Q$ :

$$
\mu \sum_{i=1}^{n} \xi_{i}^{2} \leq \sum_{i, j=1}^{n} a_{i j}(x, t) \xi_{i} \xi_{j}, \quad \mu>0
$$

where

$$
\begin{gathered}
a_{i j}=a_{j i}, \quad a_{i j}, b_{i}, a \in L^{\infty}\left(Q_{T}\right) ; \\
\frac{\partial a_{i j}}{\partial t} \in L^{1}\left(0, T ; L^{\infty}(\Omega)\right), \quad i, j, k=1, \ldots, n .
\end{gathered}
$$

It is known that the system (34), (35) admits a unique solution in $C\left([0, T] ; L^{2}(\Omega)\right) \cap H^{0,1} Q_{T}$ Ladyzhenskaja et al. (1968). Such regularity of solutions is not consistent with point models for sensors and sources involving $\delta$ functions. For example, the former require the existence of traces of the corresponding solutions on the trajectories in $\mathbb{R}^{n+1}$. On the contrary, no such difficulty will arise if one uses zone models.

Let us assume that the distributed process (34), 35 is excited at time $t=0$ by a single source $u_{0}\left(x, x^{0}\right)$ of unit intensity (see Remark 1) concentrated within some neighborhood of an unknown point $x^{0}$,

$$
\begin{aligned}
& u_{0}\left(x, x^{0}\right) \\
& =\operatorname{meas}^{-1}\left\{S_{h}\left(x^{0}\right) \cap \Omega\right\} \begin{cases}1 & \text { if } x \in S_{h}\left(x^{0}\right) \cap \Omega, \\
0 & \text { if } x \notin S_{h}\left(x^{0}\right) \cap \Omega,\end{cases}
\end{aligned}
$$

where $h>0$ is given and characterizes the effective zone $S_{h}\left(x^{0}\right)$ of the source,

$$
S_{h}\left(x^{0}\right)=\left\{x \mid\left\|x-x^{0}\right\|_{\mathbb{R}^{n}}<h\right\} .
$$

As in the above, the source localization problem is to find $x^{0}$, based on the available $m$-dimensional measurement data $y(t) \in \mathbb{R}^{m}, t \in(0, T)$, provided by $m$ given sensors,

$$
y(t)=\left(\begin{array}{c}
\int_{\Omega} \chi\left(x, S_{1}(t)\right) u(x, t) \mathrm{d} x \\
\vdots \\
\int_{\Omega} \chi\left(x, S_{m}(t)\right) u(x, t) \mathrm{d} x
\end{array}\right), \quad t \in(0, T),
$$

where

$$
\chi\left(x, S_{j}(t)\right)=\operatorname{meas}^{-1}\left\{S_{j}(t)\right\} \begin{cases}1 & \text { if } x \in S_{j}(t), \\ 0 & \text { if } x \notin S_{j}(t),\end{cases}
$$

and $S_{j}(t) \subset \Omega, \quad j=1, \ldots, r$ are the given effective sensing regions at the instant $t$. We assume that the setvalued maps $t \rightarrow S_{j}(t)$ are continuous in time with respect to the Lebesgue measure. When $S_{j}(t)=S_{j}, \quad j=$ $1, \ldots, m$, we have stationary zone observations, otherwise they are mobile.

The method of test functions described in the above for the one dimensional boundary problem (16) applies along the lines of Theorem 2 to a general system like (34 as well. Respectively, we need to introduce a control problem dual of (34) as follows (compare it with (18))

$$
\begin{gathered}
\frac{\partial z}{\partial t}=\sum_{i, j=1}^{n} \frac{\partial}{\partial x_{i}}\left(a_{i j}(x, T-t) \frac{\partial z}{\partial x_{j}}\right) \\
\quad-\sum_{i=1}^{n} \frac{\partial}{\partial x_{i}}\left(b_{i}(x, T-t) z\right)+a(x, t) z \\
+\sum_{j=1}^{m} \lambda_{j}(T-t) \chi\left(x, S_{j}(T-t)\right) \text { in } Q_{T}, \\
\left.z\right|_{\Sigma_{T}}=\left.0 \quad u\right|_{t=0}=0 .
\end{gathered}
$$

Let us introduce the set of test-functions $\Gamma$ by the formula 20 , that is,

$\Gamma=\left\{\gamma_{\lambda}=z(\cdot, T) \mid \lambda=\left(\lambda_{1}, \ldots, \lambda_{m}\right) \in\left[L^{2}(0, T)\right]^{m}\right\}$.

Then, exactly as in Sections 3 and 4 (see, e.g., (19)), we have the following system of equations to find $x^{0}$ :

$$
\begin{aligned}
\int_{\Omega} \gamma_{\lambda}(x) u_{0}\left(x, x^{0}\right) \mathrm{d} x=\int_{0}^{T} \lambda^{\prime} y(t) \mathrm{d} t, \\
\forall \lambda \in\left[L^{2}(0, T)\right]^{m} .
\end{aligned}
$$

Theorem 6. Let the set of $\Gamma$ defined in (40) be dense in $L^{2}(0,1)$ or, which is the same, the system (39) is approximately controllable in $L^{2}(0,1)$ at time $T$. Then the system (34)-(38) is ch-identifiable at time T. Namely, for an arbitrary $\varepsilon>0$ there exist $n$ test-functions $\left\{\gamma_{\lambda^{i}}, i=1, \ldots, n\right\}$ such that for any possible output $y(t), t \in(0, T)$ all solutions $x^{0} \in \Omega$ to the system (41) lie in some ball of radius $\varepsilon+2 h$. 
Let us note here that the approximate controllability of the system (39) in $L^{2}(0,1)$ was studied by Khapalov (1994(a); 1998; 2001) (see the work of Khapalov (2010) and the references therein for the case of multiplicative controls), with an algorithm on how one can construct suitable mobile supports $S_{j}(t), t \in(0, T), j=1, \ldots, m$ for sensors given.

Proof. Let $C=\left\{c_{i j}\right\}, i, j=1, \ldots, n$ be an arbitrary non-degenerate $[n \times n]$-matrix. Write

$$
\begin{aligned}
v_{i}(x)=\sum_{j=1}^{n} c_{i j} x_{j}, \quad i= & 1, \ldots, n, \\
& x=\left(x_{1}, \ldots, x_{n}\right) \in \mathbb{R}^{n} .
\end{aligned}
$$

Case 1. Assume first that all the functions $\left\{v_{i}(\cdot)\right\}$ belong to the set $\Gamma$, that is, there exist $\left\{\lambda^{(i)}(\cdot)\right\}_{i=1}^{n}$ for which

$$
v_{i}(x)=\gamma_{\lambda^{(i)}}(x), \quad i=1, \ldots, n .
$$

Then, (41) and (36) yield

$$
\begin{aligned}
& \int_{\Omega} u_{0}\left(x, x^{0}\right) \gamma_{\lambda^{(i)}}(x) \mathrm{d} x \\
&=\sum_{j=1}^{n} c_{i j} x_{j}^{0}+b_{i}\left(x^{0}\right), \quad i=1, \ldots, n
\end{aligned}
$$

where $x^{0}=\left(x_{1}^{0}, \ldots, x_{n}^{0}\right)$ and the functions $b_{i}, i=$ $1, \ldots, n$ are uniformly bounded in $\Omega$,

$$
\left|b_{i}(x)\right| \leq h\|C\|, \quad \forall x \in \Omega, \quad i=1, \ldots, n .
$$

Indeed,

$$
\begin{gathered}
\int_{\Omega} u_{0}(z, x)\left(\begin{array}{c}
\left.\gamma_{\lambda^{(1)}}(z)\right) \\
\left.\gamma_{\lambda^{(2)}}(z)\right) \\
\vdots \\
\left.\gamma_{\lambda^{(n)}}(z)\right)
\end{array}\right) \mathrm{d} z \\
=\frac{1}{\operatorname{meas}\left\{S_{h}(x) \bigcap \Omega\right\}} \int_{\substack{\left(S_{h}(x) \cap \Omega\right)-x \\
\forall x \in \Omega .}} C(z+x) \mathrm{d} z, \\
\forall x \in
\end{gathered}
$$

Hence,

$$
\begin{aligned}
& \left\|\left(b_{1}(x), \ldots, b_{n}(x)\right)\right\|_{\mathbb{R}^{n}} \\
& \quad \leq \sup _{z \in S_{h}(0)}\|C z\|_{R^{n}} \leq h\|C\|, \quad \forall x \in \bar{\Omega} .
\end{aligned}
$$

By virtue of (41) and (43), the unknown point $x^{0}$ satisfies the following system of equations:

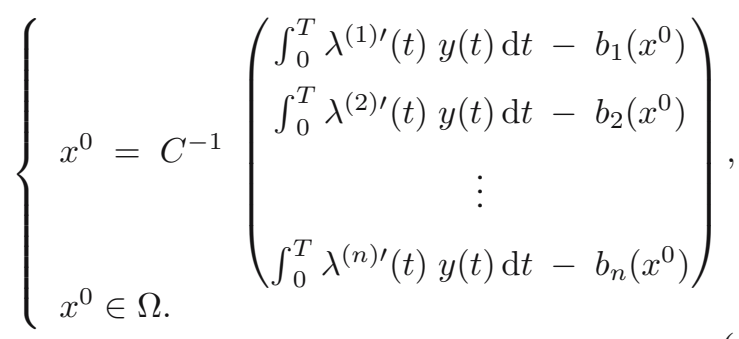

Let $x^{01}, x^{02}$ be two different solutions of (45) (if such exist, otherwise we skip the next step of the argument). Applying the estimate (44) to (45) yields

$$
\begin{aligned}
& \left\|x^{01}-x^{02}\right\|_{\mathbb{R}^{n}} \\
& \leq \sup _{x^{01}, x^{02} \in \Omega} \| C^{-1}\left(\left(b_{1}\left(x^{01}\right), \ldots, b_{n}\left(x^{01}\right)\right)^{\prime}\right. \\
& \left.\quad-\left(b_{1}\left(x^{02}\right), \ldots, b_{n}\left(x^{02}\right)\right)^{\prime}\right) \|_{\mathbb{R}^{n}} \\
& \leq 2 h\left\|C^{-1}\right\|\|C\| .
\end{aligned}
$$

The conclusion of Theorem 6 follows by choosing $C$ to be the identity matrix.

Case 2. In the general case we may not have (43). However, the assumptions of Theorem 6 provide us with such a sequence $\left\{\lambda_{k}=\left(\lambda_{k}^{(1)}, \ldots, \lambda_{k}^{(n)}\right), k=1, \ldots\right\}$ that the corresponding functions $\left\{\gamma_{\lambda_{k}^{(i)}}, i=1, \ldots, n ; k=\right.$ $1, \ldots, \infty\}$ converge in the norm of $\left[L^{2}(\Omega)\right]^{m}$ to $\left(v_{1}, \ldots, v_{n}\right)$ as in (42).

Select any $\rho>0$. Then for some $k=k^{*}$ we have

$$
\begin{array}{r}
\left|\int_{\Omega} u_{0}(z, x) \gamma_{\lambda_{k^{*}}^{(i)}} \mathrm{d} z-\int_{\Omega} u_{0}(z, x) v_{i}(z) \mathrm{d} z\right| \leq \rho \\
i=1, \ldots, n, \quad \forall x \in \Omega .
\end{array}
$$

From here, along the lines 433-445, we obtain the system

$$
\begin{aligned}
& \left\{\begin{array}{l}
x^{0}=C^{-1} \mathcal{A}, \\
x^{0} \in \Omega
\end{array}\right\}, \\
& \mathcal{A}=\left(\begin{array}{c}
c \int_{0}^{T} \lambda_{k^{*}}^{(1) \prime}(t) y(t) \mathrm{d} t-b_{1}\left(x^{0}\right)+\rho_{1}\left(x^{0}\right) \\
\int_{0}^{T} \lambda_{k^{*}}^{(2) \prime}(t) y(t) \mathrm{d} t-b_{2}\left(x^{0}\right)+\rho_{2}\left(x^{0}\right) \\
\vdots \\
\int_{0}^{T} \lambda_{k^{*}}^{(n) \prime}(t) y(t) \mathrm{d} t-b_{n}\left(x^{0}\right)+\rho_{n}\left(x^{0}\right)
\end{array}\right)
\end{aligned}
$$

for the unknown location point $x^{0}$, where

$$
\left|\rho_{i}(x)\right| \leq \rho, \quad \forall x \in \Omega, \quad i=1, \ldots, n .
$$

Thus, similarly to (46),

$$
\left\|x^{01}-x^{02}\right\|_{\mathbb{R}^{n}} \leq 2 h\left\|C^{-1}\right\|\|C\|+\varepsilon\left\|C^{-1}\right\|
$$


with

$$
\varepsilon=2 \sqrt{n} \rho .
$$

In order to ensure that $x^{01}$ and $x^{02}$ lie in a ball of radius $2 h+\varepsilon$ (as required in Theorem 6), it is sufficient to select $C$ to be the identity matrix. This completes the proof of Theorem 6.

\section{Remark 5.}

- Theorem 6 provides the formula (47) and the estimate (48) on the basis of linear test-functions. It seems plausible that this estimate can somewhat be refined by taking into account the particular shape of $\Omega$ at hand when selecting test-functions.

- Similarly to the discussion in Section 5, we can extend the results of this section to the case when the unknown source acts continuously in time.

\section{References}

Afifi, L., El Jai, A. and Merry, M. (2000). Detection and sources reconstruction in a tube, International Journal of Systems Science 31(2): 149-159.

Afifi, L., El Jai, A. and Merry, M. (2001). Regional detection and reconstruction of unknown internal or boundary sources, International Journal of Applied Mathematics and Computer Science 11(2): 319-348.

Alvez, C., Silvestre, A.L., Takahashi, T. and Tuscnak M. (2009). Solving inverse source problems using observability (with), SIAM Journal of Control and Optimization 48(3): 1632-1659.

Butkovskii, A.G. and Pustylnikov, A.M. (1987). Mobile Control of Distributed Parameter Systems, Ellis Horwood, Chichester.

Dagan, G. (1987). Theory of solute transport by groundwater, Annual Reviews of Fluid Mechanics 19: 183-215.

Dagan, G. (1989). Flow and Transport in Porous Formations, Springer-Verlag, Heidelberg, p. 465.

Demetriou, M. (2009). Centralized and decentralized policies for the containment of moving source in $2 \mathrm{D}$ diffusion processes using sensor/actuator network, 2009 American Control Conference, St. Louis, MO, USA, pp. 127-132.

Devooght, J. and Smidts, O.F. (1996). Transport of radionuclides in stochastic media: 1 . The quasi asymptotic approximation, Annals of Nuclear Energy 23(6): 499-516.

Dolecki, Sz. (1973). Observation for the one-dimensional heat equation, Studia Mathematica 48: 291-305.

Dolecki, Sz. and Russell, D.L. (1977). A general theory of observation and control, SIAM Journal of Control and Optimization 15: 185-219.

Fattorini, H.O. and Russell, D.L. (1974). Uniform bounds on biorthogonal functions for real exponentials with an application to the control theory of parabolic equations, Quarterly of Applied Mathematics 43: 45-69.
Friedman, A. (1964). Partial Differential Equations of Parabolic Type, Prentice-Hall, Englewood Cliffs, NJ.

Isakov, V. (2009). Inverse Problems for Partial Differential Equations, Springer, New York, NY, p. 360

Khapalov, A.Y. (1994a). $\quad L^{\infty}$-exact observability of the heat equation with scanning pointwise sensor, SIAM Journal Control and Optimization 32(4): 1037-1051.

Khapalov, A.Y. (1994b). Localization of unknown sources for parabolic systems on the basis of available observations, International Journal of System Sciences 25(8): 13051322.

Khapalov, A.Y. (1996). On unique continuation of the solutions of the parabolic equation from a curve, Control and Cybernetics 25(1): 451-463.

Khapalov, A.Y. (1998). Exact null-controllability for the semilinear heat equation with mobile controls of degenerate support, Technical Report Series 98-2, Mathematics Department, Washington State University, Pullman, WA

Khapalov, A.Y. (2001). Mobile point controls versus locally distributed ones for the controllability of the semilinear parabolic equation, SIAM Journal of Control and Optimization 40(7): 231-252.

Khapalov, A.Y. (2010). Controllability of Partial Differential Equations Governed by Multiplicative Controls, Lecture Notes in Mathematics, Vol. 1995, Springer, Heidelberg/Dordrecht/London/New York, NY.

Komornik, V. and Yamamoto, M. (2002). Upper and lower estimates in determining point sources in a wave equation, Inverse Problems 18(2): 319-329.

Komornik, V. and Yamamoto, M. (2005). Estimation of point sources and applications to inverse problems, Inverse Problems 21(6): 2051-2070.

Kurzhanski, A.B. and Khapalov, A.Y. (1990). Mathematical problem motivated by environmental monitoring, Proceedings of the 11th IFAC World Congress, Tallinn, Estonia, p. 5.

Ladyzhenskaja, O.H., Solonnikov, V.A. and Ural'ceva, N.N. (1968). Linear and Quasi-linear Equations of Parabolic Type, AMS, Providence, RI.

Matthes, J., Gruell, L. and Keller, H. (2005). Source localization by spatially distributed electronic noses for advection and diffusion, IEEE Transactions on Signal Processing 53(5): 1711-1719.

Nicaise, S. and Zair, O. (2004). Determination of point sources in vibrating beams by boundary measurements: Identifiability, stability, and reconstruction results, Electronic Journal of Differential Equations (20): 1-17.

PAGIS (1989). Performance Assessment of Geological Isolation Systems for Radioactive Waste-Summary, Nuclear Science and Technology, Joint Research Centre, EUR 11775, $11776 \mathrm{EN}$.

Puel, J.-P. and Yamamoto, M. (1995). Applications de la controlabilite exacte à quelques problèmes inverses hyperboliques, Computer Rendus de l'Académie des Sciences Serie 1, Mathématique 320(10): 1171-1176. 
Saut, J.-C. and Scheurer, B. (1987). Unique continuation for some evolution equations, Journal of Differential Equations 66(1): 118-139.

Seinfeld, J.H. (1986), Atmospheric Chemistry and Physics of Pollution, Wiley Interscience, Somerset, NJ.

Shukla, J.B., Hallam T. G. and Capasso V. (Eds.) (1987). Mathematical Modelling of Environmental and Ecological Systems, Elsevier, Amsterdam.

Sivergina, I.F., Polis, M.P. and Kolmanovsky, I. (2003). Source identification for parabolic equations, Mathematics of Control, Signals, and Systems 16(2-3): 141-157.

Tzanos, P. and Zefran, M. (2006). Stability analysis of information based control for biochemical source localization, 2006 IEEE International Conference on Robotics and Automation, Orlando, FL, USA, pp. 3116-3121.

Uciński, D. (2000). Optimal sensor location for parameter estimation of distributed processes, International Journal of Control 73(13): 1235-1248.

Uciński, D. (2005). Optimal Measurement Methods for Distributed Parameter System Identification, CRC Press, Boca Raton, FL.

Wang, Y. H. (2007). Global uniqueness and stability for an inverse plate problem, Journal of Optimization Theory and Applications 132(1): 161-173.
Williams, M.M.R. (1992). A new model for describing the transport of radionuclides through fractured rocks, Annals of Nuclear Energy 19(10-12): 791-824.

Williams, M.M.R. (1993). Radionuclides transport through fractured rocks, Annals of Nuclear Energy 20(3): 279-297.

Yamamoto, M. (1995). Stability, reconstruction formula and regularization for an inverse source hyperbolic problem by a control method, Inverse Problems 11(2): 481-496.

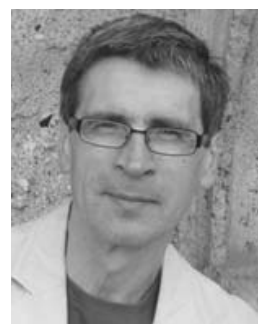

Alexander Khapalov received his Ph.D. in mathematics from the Institute of Mathematics and Mechanics of the Russian Academy of Sciences (Yekaterinburg, Russia) in 1982. Besides the aforementioned institute, he has held positions at the International Institute for Applied System Analysis in Laxenburg, Austria; at Oregon State University, USA; and he is currently with the Department of Mathematics at Washington State University, USA. His current research interests focus on swimming models and their motion capabilities, controllability of linear and nonlinear PDEs governed by multiplicative controls, smart materials, point sensors and actuators including mobile ones, and the granular material formation problem.

Received: 16 July 2009

Revised: 11 March 2010

Re-revised: 3 May 2010 